\title{
Caveolin and lipid domains-close companions in managing cellular pathways
}

\author{
Jody V. Vykoukal ${ }^{1,2} \cdot$ Johannes F. Fahrmann ${ }^{1,2} \cdot$ Timothy C. Thompson $^{3}$ \\ Published online: 16 May 2020 \\ (C) Springer Science+Business Media, LLC, part of Springer Nature 2020
}

Identification of lipid rafts (highly dynamic plasma membrane domains enriched in cholesterol and sphingolipids) and caveolae, a subdomain of lipid rafts, established a foundation for a remarkable period of multidisciplinary research on the molecular and cellular functions of these structures [1, 2]. One branch of research that evolved rapidly from these findings focused on caveolin, in particular caveolin-1 (Cav1), a caveola coat protein that is required for caveola formation, and demonstrates specific scaffolding functions [3]. One of the most important meetings in the history of caveolae and caveolin research was an NCI-sponsored workshop organized by Suresh Mohla in 2002. The proceedings of this meeting documented the amazing breadth of caveolae/caveolin function, and their profound impact on biological activities. The initiative and organization of this meeting were truly prescient as shown by the overall report of the meeting topics addressed during the meeting, which included lipid raft structure, molecular trafficking by caveolae, caveolae-mediated cell signaling, and caveolin function in malignancy [4]. The collection of papers included in the special issue on Caveolin and Lipid Domains in Cancer consolidate the current molecular and cellular biological information regarding caveolin, lipid domains, and their functional interactions, and establish a benchmark for progress in this area of research.

Studies of the molecular interactions of Cav1 and lipid domains in cancer have led to major advances in understanding the underlying mechanisms of malignant behavior of many cancers, and substantial progress in cancer treatment.

Timothy C. Thompson

timthomp@mdanderson.org

1 Department of Clinical Cancer Prevention, The University of Texas MD Anderson Cancer Center, Houston, TX, USA

2 The McCombs Institute for the Early Detection and Treatment of Cancer, The University of Texas MD Anderson Cancer Center, Houston, TX, USA

3 Department of Genitourinary Medical Oncology, The University of Texas MD Anderson Cancer Center, Houston, TX, USA
For example, phosphorylation of Cav1 on tyrosine-14 (a major Src kinase target) was shown to regulate focal adhesion tension, potentially promoting cancer cell motility through interactions with the highly conserved caveolin scaffolding domain [5]. There has also been substantial progress toward caveolae/Cav1-based predictive biomarkers and drug targeting. Examples of recent advances include novel findings that show caveolae/Cav1 is a predictive biomarker for nabpaclitaxel and other albumin-based cancer therapeutics [6]. This study documented the importance of caveolae-mediated endocytosis in albumin uptake and response to albuminbound chemotherapy. In addition, it was shown that Cav1 regulates HER-2 half-life and availability at the cell membrane. This regulation affected trastuzumab binding and therapeutic efficacy in preclinical studies [7].

Recent studies document the importance of caveolae/Cav1 in cancer cell metabolism. In particular, Cav1 regulation of lipid metabolic pathways is noteworthy, given the expansive intracellular regulation of lipid trafficking and metabolic control conferred by Cav1 in specific cancers. Soluble Cav1 exists in lipoprotein-like particles functioning as a lipid chaperone that supports cellular lipid import, export, and trafficking through interactions with various lipids including cholesterol and sphingolipid fatty acid domains [8]. Subcellular distribution of Cav1 is responsive to phosphorylation by upstream oncogenic serine and tyrosine kinases and can effect context-dependent integration and regulation of metabolic resources necessary for malignant progression [5, 9]. The association of Cav1 and metabolic reprogramming in cancer likely involves selection for altered intracellular rewiring and reprogramming that is enabled by the unique properties of Cav1, including vast capacity for subcellular compartmentalization, trafficking and scaffolding functions, and regulation by oncogenic activities. It is conceivable that continued progress in understanding the role of Cav1 in lipid metabolism will reveal metabolic vulnerabilities amenable to direct targeting as anticancer therapy. Progress in our understanding of Cav1 from eponymous scaffolding protein to essential phenotypic mediator with roles in molecular trafficking, cell signaling, 
and network-level orchestration of metabolic programs has yielded more integrated understanding and mechanistic knowledge of the essential and diverse roles of Cav1 that opens broad new opportunities for beneficial application in cancer biology and medicine.

\section{References}

1. Simons, K., \& Ikonen, E. (1997). Functional rafts in cell membranes. Nature, 387(6633), 569-572.

2. Brown, D. A. (1998). Functions of lipid rafts in biological membranes. Ann Rev Cell Dev Biol, 14, 111-136.

3. Glenney Jr., J. R. (1989). Tyrosine phosphorylation of a 22-kDa protein is correlated with transformation by Rous sarcoma virus. $J$ Biol Chem, 264(34), 20163-20166.

4. Carver, L. A., Schnitzer, J. E., Anderson, R. G., \& Mohla, S. (2003). Role of caveolae and lipid rafts in cancer: workshop summary and future needs. Cancer Res, 63, 6571-6574.
5. Meng, F., Saxena, S., Liu, Y., Joshi, B., Wong, T. H., Shankar, J., Foster, L. J., Bernatchez, P., \& Nabi, I. R. (2017). The phosphocaveolin-1 scaffolding domain dampens force fluctuations in focal adhesions and promotes cancer cell migration. Mol Biol Cell, 28, 2190-2201.

6. Chatterjee, M., et al. (2017). Caveolae-mediated endocytosis is critical for albumin cellular uptake and response to albumin-bound chemotherapy. Cancer Res, 77(21), 5925-5937.

7. Pereira, P. M. R., et al. (2018). Caveolin-1 mediates cellular distribution of HER2 and affects trastuzumab binding and therapeutic efficacy. Nat Comm, 9, 5137.

8. Liu, P., Rudick, M., \& Anderson, R. G. W. (2002). Multiple Functions of Caveolin-1. J Biol Chem, 277, 41295-41298.

9. Hau, A. M., et al. (2019). Dynamic regulation of caveolin-1 phosphorylation and caveolae formation by mammalian target of rapamycin complex 2 in bladder cancer cells. Am Journal Pathol, 189(9), 1846-1862.

Publisher's note Springer Nature remains neutral with regard to jurisdictional claims in published maps and institutional affiliations. 